\title{
Agnathia-otocephaly complex diagnosed by prenatal ultrasound: a case report
}

\author{
Xueqin Ji, Yuan Zhao, Yan Xia, Yang Wu, Rui Xu, Hairui Wang, Feihai Liyan \\ Department of Ultrasound, Ningxia Hui Autonomous Region Maternity and Child Health Hospital \& Ningxia Children's Hospital, Yinchuan, China \\ Correspondence to: Yuan Zhao. Department of Ultrasound, Ningxia Hui Autonomous Region Maternity and Child Health Hospital \& Ningxia \\ Children's Hospital, No. 127, Hupan Road, Jinfeng District, Yinchuan 750011, China. Email: 17398441230@163.com.
}

\begin{abstract}
Agnathia-otocephaly complex (AOC) is a rare and complex craniofacial malformation characterized by mandibular hypoplasia or agnathia, auricular fusion (synotia), and microstomia with oroglossal hypoplasia or aglossia. It can occur alone or in combination with forebrain anomalies and cardiac malformations and has an extremely poor prognosis. Here, we report a case of AOC diagnosed by systemic fetal screening at a gestational age of $25^{+4}$ weeks. Ultrasound revealed that the S-curve formed by the normal lower jaw and lower lip had disappeared, the lower jaw and mandible were invisible, the mouth was extremely small, and the oral fissure was "pinhole-shaped". There was a cone-shaped perioral bulge. Both ears were located in the front side of the neck, and the right foot was inverted. Excessive amniotic fluid was observed. The absence of a mandible was confirmed on X-ray examination after induced abortion. Specimen observation showed that the ear positions were extremely low, and both earlobes were connected in the front side of the neck. It was particularly challenging to identify the development of the mandible and locate auricles during prenatal ultrasound diagnosis, and the prenatal diagnosis of AOC was confirmed by combining two-dimensional and three-dimensional ultrasound in our current case.
\end{abstract}

Keywords: Agnathia-otocephaly complex (AOC); prenatal diagnosis; three-dimensional ultrasound; case report

Submitted Mar 26, 2021. Accepted for publication Aug 17, 2021.

doi: $10.21037 / \mathrm{tp}-21-235$

View this article at: https://dx.doi.org/10.21037/tp-21-235

\section{Introduction}

Agnathia-otocephaly complex (AOC) is a rare congenital malformation complex of the first pharyngeal arch, with an incidence of about 1/70,000 births (1). Its clinical manifestations include mandibular hypoplasia or agnathia, auricular fusion (synotia), and microstomia with oroglossal hypoplasia or aglossia. AOC can occur alone or in combination with holoprosencephaly, urinary tract anomalies, and cardiac anomalies (2). Here, we report a case of AOC that was detected and diagnosed by prenatal ultrasound. We present the following article in accordance with the CARE reporting checklist (available at https:// dx.doi.org/10.21037/tp-21-235).

\section{Case presentation}

A 30-year-old female with menopause for $25^{+4}$ weeks presented to our center. She was previously healthy, with regular menstruation before pregnancy. She denied any history of infection, radiation, or chemical toxicant exposure during the pregnancy. She did not receive any obstetric examination in her first trimester. Her blood pressure and blood glucose were normal. There was no vaginal bleeding or spotting, and no fertility preservation treatment had been applied. Systemic ultrasound of the fetus revealed a biparietal diameter (BPD) of $6.2 \mathrm{~cm}$, a head circumference (HC) of $23.1 \mathrm{~cm}$, an abdominal circumference (AC) of $20.8 \mathrm{~cm}$, and a femur length (FL) of $5.9 \mathrm{~cm}$. The maximal depth of amniotic fluid was $9.3 \mathrm{~cm}$. The fetal heart rate was 160 beats/min, and a regular arrhythmia was present. The umbilical artery (UA) systolic/diastolic (S/D) ratio was 2.45 . The fetus had a left occiput anterior (LOA) position.

The S-curve formed by the normal lower jaw and lower lip had disappeared, and the lower jaw and mandible were 

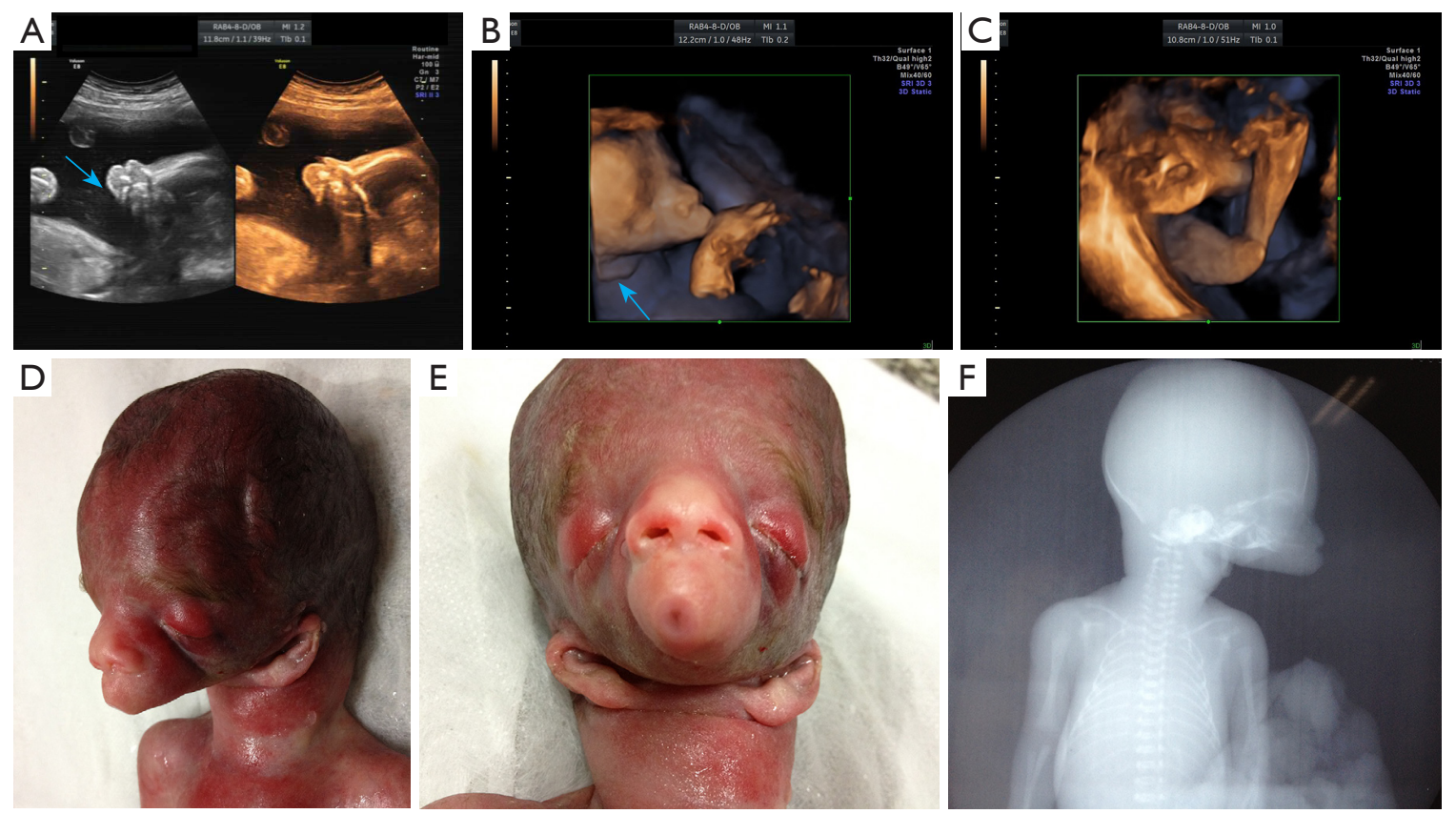

Figure 1 Ultrasound findings of the AOC fetus at 25+ weeks and after induced abortion. (A) The lower jaw and mandible are absent on the sagittal section (arrows); (B) volumetric 3D imaging of fetal face shows a tubular bulge in the lower part of the face, a small mouth, and auricles located in the front side of the neck (arrows); (C) right foot inversion; (D) the lateral view of the face of the induced fetus shows the absence of the lower jaw; (E) the frontal view of the face of the induced fetus shows bilateral ptosis, small mouth, absence of lower jaw, and otocephaly; (F) X-ray of the induced fetus shows the absence of the mandible. This image is published with the mother's consent.

not visible (Figure $1 A$ ). The mouth was extremely small, and the oral fissure was "pinhole-shaped." There was a coneshaped perioral bulge. Both ears were located on the front side of the neck (Figure 1B). The long-axis view of the right calf also showed plantar echogenicity (Figure 1C), and there was no change in posture during the 30-minute dynamic observation. The placenta was grade 0 , measured $2.3 \mathrm{~cm}$ thick, and attached to the anterior uterus wall. Ultrasound suggested a single live intrauterine fetus in cephalic presentation. The fetal facial developmental abnormalities included congenital agnathia, right foot inversion, and polyhydramnios.

The pregnant woman and her family refused chromosomal examination and insisted on induced abortion. A stillborn male baby weighing $854 \mathrm{~g}$ was delivered. Postinduction examination showed a tubular periorbital bulge (Figure 1D), bilateral ptosis, small mouth, lip hypoplasia (Figure 1E), and a marked absence of the lower jaw. The auricles were inferiorly displaced, and both ears were located anteriorly and connected to the neck (Figure $1 E$ ). The right foot was inverted. $\mathrm{X}$-ray examination further showed the absence of the mandible (Figure $1 F$ ) and inversion of the right foot. The patient's medical treatment process has been described in timeline (Figure 2). Written informed consent was obtained from the patient for publication of this case report and accompanying images. A copy of the written consent is available for review by the editorial office of this journal. All procedures performed in studies involving human participants were in accordance with the ethical standards of the institutional and/or national research committee(s) and with the Helsinki Declaration (as revised in 2013).

\section{Discussion}

The etiology of AOC remains unknown but is believed to be associated with genetic and environmental factors. Lin et al. (3) suggested that AOC arises from defects in the ventral portion of the first branchial arch, which are often caused by the poor neural crest cell migration in the early embryonic stage or the premature proliferation of the embryonic disc. Agnathia has been reported to be associated with the genes OTX2, PRRX1, and CRKL $(4,5)$. OTX2 is a transcription factor; it is expressed in 


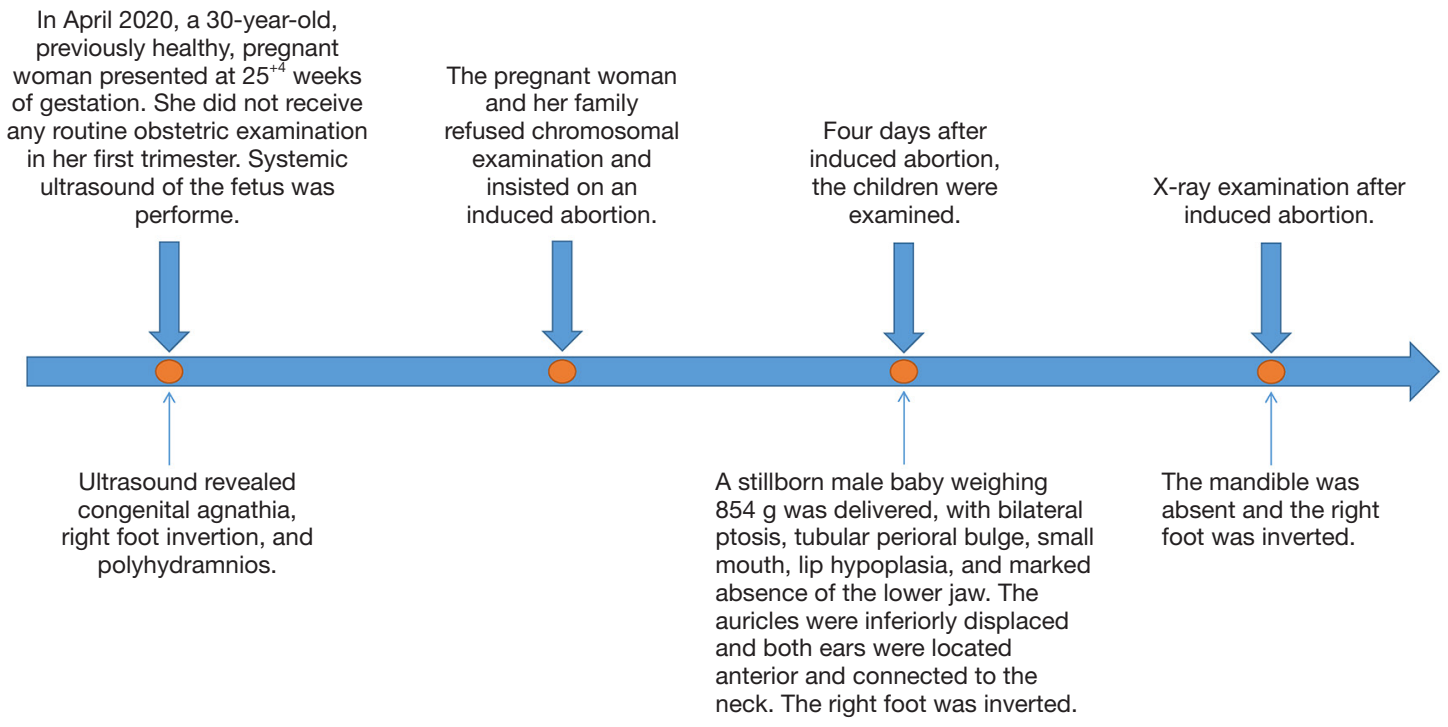

Figure 2 Timeline of diagnosis and treatment.

the mesenchymal cells of the midbrain head and neural crest cells and distributed in the mandibular region; it has been proved to cause craniofacial malformation and holoprosencephaly. PRRX1 is associated with the expressions of cranial mesenchyme in the maxilla, frontal sinus, and mandibular prominence. In addition, some related family studies show that the disease may have autosomal dominant and recessive inheritance patterns. In addition to genetic factors, theophylline, salicylic acid, and other drugs (6) will increase disease risk.

Fetal facial development occurs primarily during the fifth to eighth weeks of gestation. The fetal mandible develops from the mandibular arch of the first branchial arch. The first pair of branchial clefts develop into the external acoustic meatus. The mesenchyme around the branchial clefts proliferates and develops into auricles, which are initially low and then gradually pushed posteriorly and superiorly with the development of the mandible and neck. The first pair of branchial arches is also involved in developing the tongue, the first and second pairs in developing the anterior two-thirds of the tongue, and the second, third, and fourth pairs in the development of the posterior third the tongue. When the first branchial arch is extremely dysplastic, the mandible may not develop or even be absent, which is often accompanied by synotia and microstomia; as a result, the mouth becomes a cavity or even a blind tube that is not connected to the pharynx. Tongue dysplasia will occur, and there may be a tiny posterior tongue or no tongue at all.
Prenatal ultrasound is an effective tool to screen for and diagnose AOC. It is particularly challenging to identify the development of the mandible and locate auricles during prenatal ultrasound diagnosis. Prenatal diagnosis of the disease is difficult $(1,7,8)$. The mandible can be screened on the sagittal and transverse sections of the face. In this case, we found that the normal S-shaped curve formed by the mandible and the lower lip disappears, and the lower jaw and the mandible are absent. In order to get more facial image information, we supplemented the three-dimensional ultrasound examination and found that the fetal lower part was tubular uplift, small mouth, and other features, and showed the auricle in front of the neck, which is difficult to show in a two-dimensional ultrasound. Combined with the characteristics of two-dimensional ultrasound and threedimensional ultrasound, we can diagnose non-mandibular ear deformity. The face of the induced labor fetus showed ptosis, a small mouth, no mandible, and ear deformity. We found that this is highly consistent with the threedimensional craniofacial structure displayed by prenatal three-dimensional ultrasound volume imaging, which fully shows the advantages of three-dimensional ultrasound in diagnosing non-mandibular ear deformity. It can intuitively and stereoscopically display the characteristics of facial deformity, especially in the detection of ear deformity. When two-dimensional ultrasound found abnormal facial contour and suspected the existence of the disease, it is recommended to combine three-dimensional ultrasound for diagnosis and evaluation. 
At present, most of the patients are diagnosed in the second and third trimesters of pregnancy. Prenatal ultrasound screening in the first trimester of pregnancy found that fetal facial contour abnormalities, mandibular loss, or mandibular dysplasia are the key to diagnosing this disease. Three-dimensional ultrasound technology can provide more detailed information on craniofacial deformity, which is helpful for more accurate diagnosis and evaluation of the disease.

The prognosis of AOC fetuses is extremely poor, and long-term survival is practically impossible (9). A small number of isolated born-alive infants will die of respiratory distress due to the absence of the mandible hindering the development of adjacent structures, which often involves the naso-mandibular complex and the oropharynx, resulting in respiratory distress, feeding difficulties, and speech and/ or hearing impairment (10). Those who survive still require tracheostomy for assisted breathing and a gastrostomy tube for assisted feeding (11), with daily communication depending on nonverbal means. Mandibular distraction osteogenesis can be performed to improve the local facial appearance and a few other functions.

When an abnormal facial structure is found in the prenatal examination, further genetic counseling and detailed systematic evaluation should be provided. Considering the factors of abnormal deformity caused by a gene mutation in the mandible and ear deformity, it is suggested to carry out gene detection when suspected of the disease. New sequencing techniques such as whole exon group and whole-genome sequencing can improve the diagnosis rate of this rare disease. Some studies have proved that exome sequencing clarifies the fetal molecular pathology of this disease (12). At the same time, careful examination of other systems is needed to assess whether the structural deformities of other systems are incorporated. When diagnosing no mandibular ear malformation, we should conduct a detailed fetal chromosome diagnosis and detailed evaluation of other system organs, fully inform the parents, follow the parents' wishes, timely diagnosis, and follow-up treatment.

In our case, 2D and 3D ultrasound were applied in the diagnosis of AOC. However, ultrasound-based examination of the tongue and ear canal is difficult due to their complex anatomy, and therefore further investigations are warranted.

In conclusion, when a prenatal ultrasound reveals the absence of the lower jaw and mandible, a small mouth, and extremely low ear position, the combination of $2 \mathrm{D}$ ultrasound with $3 \mathrm{D}$ ultrasound can be performed to confirm a potential diagnosis of AOC.

\section{Acknowledgments}

We thank Simin Zhang for the technical assistance. Funding: None.

\section{Footnote}

Reporting Checklist: The authors have completed the CARE reporting checklist. Availabe at https://dx.doi.org/10.21037/ tp-21-235

Conflicts of Interest: All authors have completed the ICMJE uniform disclosure form (available at https://dx.doi. org/10.21037/tp-21-235). The authors have no conflicts of interest to declare.

Ethical Statement: The authors are accountable for all aspects of the work in ensuring that questions related to the accuracy or integrity of any part of the work are appropriately investigated and resolved. Written informed consent was obtained from the patient for publication of this case report and accompanying images. A copy of the written consent is available for review by the editorial office of this journal. All procedures performed in studies involving human participants were in accordance with the ethical standards of the institutional and/or national research committee(s) and with the Helsinki Declaration (as revised in 2013).

Open Access Statement: This is an Open Access article distributed in accordance with the Creative Commons Attribution-NonCommercial-NoDerivs 4.0 International License (CC BY-NC-ND 4.0), which permits the noncommercial replication and distribution of the article with the strict proviso that no changes or edits are made and the original work is properly cited (including links to both the formal publication through the relevant DOI and the license). See: https://creativecommons.org/licenses/by-nc-nd/4.0/.

\section{References}

1. Faye-Petersen O, David E, Rangwala N, et al. Otocephaly: report of five new cases and a literature review. Fetal Pediatr Pathol 2006;25:277-96.

2. Schiffer C, Tariverdian G, Schiesser M, et al. Agnathiaotocephaly complex: report of three cases with 
involvement of two different Carnegie stages. Am J Med Genet 2002;112:203-8.

3. Lin HH, Liang RI, Chang FM, et al. Prenatal diagnosis of otocephaly using two-dimensional and threedimensional ultrasonography. Ultrasound Obstet Gynecol 1998;11:361-3.

4. Herman S, Delio M, Morrow B, et al. Agnathia-otocephaly complex: a case report and examination of the OTX2 and PRRX1 genes. Gene 2012;494:124-9.

5. Gekas J, Li B, Kamnasaran D. Current perspectives on the etiology of agnathia-otocephaly. Eur J Med Genet 2010;53:358-66.

6. Wai LT, Chandran S. Cyclopia: isolated and with agnathia-otocephaly complex. BMJ Case Rep 2017;2017:bcr2017220159.

7. Diep J, Kam D, Munir F, et al. Otocephaly Complex: Case Report, Literature Review, and Ethical Considerations. A A Case Rep 2016;7:44-8.

8. Jagtap SV, Saini N, Jagtap S, et al. Otocephaly: AgnathiaMicrostomia-Synotia Syndrome- A Rare Congenital

Cite this article as: Ji X, Zhao Y, Xia Y, Wu Y, Xu R, Wang H, Liyan F. Agnathia-otocephaly complex diagnosed by prenatal ultrasound: a case report. Transl Pediatr 2021;10(8):2131-2135. doi: $10.21037 / \mathrm{tp}-21-235$
Anomaly. J Clin Diagn Res 2015;9:ED03-ED4.

9. Golinko MS, Shetye P, Flores RL, et al. Severe AgnathiaOtocephaly Complex: Surgical Management and Longitudinal Follow-up From Birth Through Adulthood. J Craniofac Surg 2015;26:2387-92.

10. Bixler D, Ward R, Gale DD. Agnathia-holoprosencephaly: a developmental field complex involving face and brain. Report of 3 cases. J Craniofac Genet Dev Biol Suppl 1985;1:241-9.

11. Gonzalez SR, Jones JK, Golinko MS. Surgical Approach in a Patient With Agnathia-Otocephaly Complex: ThreeStage Mandibular Distraction Protocol. J Craniofac Surg 2020;31:e84-e89.

12. Sergouniotis PI, Urquhart JE, Williams SG, et al. Agnathia-otocephaly complex and asymmetric velopharyngeal insufficiency due to an in-frame duplication in OTX2. J Hum Genet 2015;60:199-202.

(English Language Editor: J. Gray) 